\title{
A NEW EFFICIENT FPGA DESIGN OF RESIDUE-TO-BINARY CONVERTER
}

\author{
Edem Kwedzo Bankas and Kazeem Alagbe Gbolagade \\ Department of Computer Science, Faculty of Mathematical Science, \\ University for Development Studies, Ghana
}

\begin{abstract}
In this paper, we introduce a new $6 n$ bit Dynamic Range Moduli set $\left\{2^{2 n}, 2^{2 n}+1,2^{2 n}-1\right\}$ and then present its associated novel reverse converters. First, we simplify the Chinese Remainder Theorem in order to obtain an efficient reverse converter which is completely memory less and adder based. Next, we present a low complexity implementation that does not require the explicit use of modulo operation in the conversion process and we demonstrate that theoretically speaking it outperforms state of the art equivalent reverse converters. We also implemented the proposed converter and the best equivalent state of the art reverse converters on Xilinx Spartan 6 FPGA. The experimental results confirmed the theoretical evaluation. The FPGA synthesis results indicate that, on the average, our proposal is about $52.35 \%$ and $43.94 \%$ better in terms of conversion time and hardware resources respectively.
\end{abstract}

\section{KEYWORDS}

Dynamic Range, reverse converters, Field programmable gate arrays, Residue Arithmetic, Chinese Remainder Theorem

\section{INTRODUCTION}

The use of alternative number systems in the implementation of application specific Digital Signal Processing (DSP) systems has gained a remarkable importance in recent years. This is due to the carry propagation problem associated with the conventional number system such as the binary numbers. The attractive carry free properties of Residue Number System (RNS) makes it a suitable candidate. RNS is an integer system which has the potential for high speed and parallel computations. RNS is mostly applied in addition and multiplication dominated DSP applications such as Digital Filtering and Convolutions [1]. In RNS, arithmetic operations such addition, subtraction, and multiplication can be carried out independently and concurrently in several residue channels more efficiently than in the conventional binary system [2].

The difficult RNS arithmetic operations include: division, magnitude comparison, overflow detection, sign detection, moduli selection and reverse conversion. For a successful RNS implementation, moduli selection and reverse conversion are the most critical issues. Reverse conversion has become an important research topic as the solution to the other RNS arithmetic operations depend largely on conversion. In literature, moduli sets that have been presented are

DOI : 10.5121/vlsic.2013.4601 
International Journal of VLSI design \& Communication Systems (VLSICS) Vol.4, No.6, December 2013

classified according to the number of channels or their lengths and their Dynamic Range (DR). Length-3 moduli sets such as $\left\{2^{\mathrm{n}}, 2^{\mathrm{n}}-1,2^{\mathrm{n}}+1\right\}$ [3], [4], [5], $\left\{2^{\mathrm{n}}, 2^{\mathrm{n}}-1,2^{\mathrm{n}-1}-1\right\}[6],[7],\left\{2^{\mathrm{n}}, 2^{\mathrm{n}+1}-\right.$ $\left.1,2^{\mathrm{n}}-1\right\}$ [8], [9] have been investigated extensively. Subsequently, other moduli sets with larger DR have been introduced, examples of which are $\left\{2^{2 \mathrm{n}+1}-1,2^{\mathrm{n}}, 2^{\mathrm{n}}-1\right\}[10],\left\{2^{2 \mathrm{n}}, 2^{2 \mathrm{n}+1}-1,2^{\mathrm{n}}-\right.$ $1\}$ [11], $\left\{2^{2 \mathrm{n}+1}-1,2^{2 \mathrm{n}+1}, 2^{2 \mathrm{n}}-1\right\}[12]$ and $\left\{2^{\mathrm{n}}, 2^{2 \mathrm{n}}-1,2^{2 \mathrm{n}}+1\right\}[13]$.

Several reverse conversion techniques have been proposed based on either the Chinese Remainder Theorem (CRT) [14], [15], [16], or the Mixed Radix Conversion (MRC) [17]. The major CRT problem is the complex and slow modulo-M operation $\left(\mathrm{M}=\mathrm{m}_{1} \mathrm{~m}_{2} \mathrm{~m}_{3} \ldots \mathrm{m}_{\mathrm{n}}\right.$ being the system dynamic range, thus a rather large constant to deal with).

In this paper, we introduce a novel moduli set $\left\{2^{2 \mathrm{n}}, 2^{2 \mathrm{n}}+1,2^{2 \mathrm{n}}-1\right\}$ by enhancing the modulus $2^{\mathrm{n}}$ to $2^{2 \mathrm{n}}$ in $\left\{2^{\mathrm{n}}, 2^{2 \mathrm{n}}+1,2^{2 \mathrm{n}}-1\right\}$ [13]. Next, we present an efficient reverse converter based on the CRT which results in efficient VLSI architecture design with high speed conversion and low cost hardware requirements. Theoretically, our proposal outperforms equivalent state of the art converters. We also implemented the proposed converter and the best equivalent state of the art converters on Xilinx Spartan 6 FPGA. The synthesis results are given in terms of the number of slices and input-to-output gate delays in nano seconds. The results indicate that, on the average, our proposal is about $52.35 \%$ and $43.94 \%$ better than existng equivalent state of the art in terms of conversion time and hardware resource utilization respectively.

The rest of the paper is structured as follows. Section 2 provides a brief background information on reverse conversion. In Section 3, the novel moduli set is introduced, and the associated reverse conversion algorithm is presented. The hardware implementation of the proposed algorithm is described in Section 4, and Section 5 evaluates the performance of the proposed scheme. Finally, the paper is concluded in Section 6.

RNS is defined in terms of a set of relatively prime moduli set $\left\{m_{i}\right\}_{i=1, k}$, such that $\operatorname{gcd}\left(m_{i}, m_{j}\right)=1$ for $\mathrm{i} \neq \mathrm{j}$, where $\operatorname{gcd}\left(\mathrm{m}_{\mathrm{i}}, \mathrm{m}_{\mathrm{j}}\right)$ means the greatest common divisor of $\mathrm{m}_{\mathrm{i}}$ and $\mathrm{m}_{\mathrm{j}}$, while $M=\prod_{i=1}^{k} m_{i}$, is the dynamic range. The residues of a decimal number $\mathrm{X}$ can be derived as $\mathrm{x}_{\mathrm{i}}$ $=|X|_{\mathrm{mi}}$ denotes $\mathrm{X} \bmod \mathrm{m}_{\mathrm{i}}$ operation.

The main methods for reverse conversion are based on the CRT, New CRT and MRC techniques. In this paper, we utilizes the CRT. Given a moduli set $\left\{\mathrm{m}_{\mathrm{i}}\right\}_{\mathrm{i}=1,3}$, the residues $\left(\mathrm{x}_{1}, \mathrm{x}_{2}, \mathrm{x}_{3}\right)$ can be converted into the corresponding decimal number $\mathrm{X}$ using the CRT as follows [2] :

$$
X=\left.\left.\left|\sum_{i=1}^{k} m_{i}\right| M_{i}^{-1}\right|_{m_{1}} x_{i}\right|_{M}
$$

Where $M=\prod_{i=1}^{k} m_{i}, M_{i}=\frac{M}{m_{i}}$ and $M_{i}^{-1}$ is the multiplicative inverse of $\mathrm{M}_{\mathrm{i}}$ with respect to ( w.r.t ) $\mathrm{m}_{\mathrm{i}}$

The complexity of Equation [1] is significantly reduced by using the proposed moduli set $\left\{2^{2 \mathrm{n}}\right.$, $\left.2^{2 \mathrm{n}}+1,2^{2 \mathrm{n}}-1\right\}$. 
International Journal of VLSI design \& Communication Systems (VLSICS) Vol.4, No.6, December 2013

\section{NeW Moduli SET WiTh Proposed REVERSE CONVERTER}

For a given RNS moduli set to be legitimate, it is required that all the elements in the set to be co-prime. Thus, in order to prove that the proposed set can be utilized for the construction of valid RNS architecture, we have to demonstrate that the moduli $2^{2 \mathrm{n}}, 2^{2 \mathrm{n}}+$ 1 and $2^{2 \mathrm{n}}-1$ are pair-wise relatively prime.

Theorem 1. The moduli $2^{2 n}, 2^{2 n}+1$, and $2^{2 n}-1$ are pair-wise relatively prime numbers.

Proof. :

Since $2^{2 n}+1$, and $2^{2 n}-1$ have been already shown to be relatively prime by [13], we only need to demonstrate that $2^{2 n}$ is coprime to $2^{2 n}+1$, and $2^{2 n}-1$. From the Euclidean theorem, we have $\operatorname{gcd}(a, b)=\operatorname{gcd}\left(b,|a|_{b}\right)$. Therefore, $\operatorname{gcd}\left(2^{2 n}, 2^{2 n}+1\right)=\operatorname{gcd}\left(2^{2 n}+1,\left|2^{2 n}\right|_{2^{2 n}+1}\right)=\operatorname{gcd}\left(2^{2 n},-1\right)=1$. Similarly, $\operatorname{gcd}\left(2^{2 n}, 2^{2 n}-1\right)=\operatorname{gcd}\left(2^{2 n}-1,\left|2^{2 n}\right|_{2^{2 n}-1}\right)=\operatorname{gcd}\left(2^{2 n}, 1\right)=1$. The numbers $2^{2 n}, 2^{2 n}+1$, and $2^{2 n}-1$ are pairwise relatively prime because all the greatest common divisors are 1 .

Theorem 2. Given that the moduli set $\left\{m_{1}, m_{2}, m_{3}\right\}$ with $m_{1}=2^{2 n}, m_{2}=$ $2^{2 n}+1$, and $m_{3}=2^{2 n}-1$ the following holds true:

$$
\begin{gathered}
\left|\left(m_{2} m_{3}\right)^{-1}\right|_{m_{1}}=-1 \\
\left|\left(m_{1} m_{3}\right)^{-1}\right|_{m_{2}}=-2^{2 n-1} \\
\left|\left(m_{1} m_{2}\right)^{-1}\right|_{m_{3}}=2^{2 n-1}
\end{gathered}
$$

Proof. :

If it can demonstrated that $\left|\left(2^{n}+1\right) \times\left(2^{n}-1\right) \times-1\right|_{2^{2 n}}=1$, then -1 is the multiplicative inverse of $\left(2^{n}+1\right) \times\left(2^{n}-1\right)$ with respect to $2^{2 n}:\left|\left(2^{4 n-1}\right) \times-1\right|_{2^{2 n}}=$ 1. Similarly, $\left|\left(2^{2 n}\right) \times\left(2^{2 n}-1\right) \times\left(-2^{2 n-1}\right)\right|_{2^{2 n}+1}=\left|(2) \cdot\left(2^{4 n-1}\right)\right|_{2^{2 n}+1}=1$. Agair. $\left|\left(2^{2 n}\right) \times\left(2^{2 n}+1\right) \times\left(2^{2 n-1}\right)\right|_{2^{2 n-1}}=\left|(2) \cdot\left(2^{4 n-1}\right)\right|_{2^{2 n-1}}=1$.

The relation below is utilized in the subsequent theorem: Given the moduli set $\left\{m_{1}, m_{2}, m_{3}\right\}$ with $\mathrm{m}_{1}=2^{2 n}, m_{2}=2^{2 n}+1$, and $m_{3}=2^{2 n}-1$, the relation below holds true:

$$
m_{2} m_{3}=m_{1}^{2}-1
$$


International Journal of VLSI design \& Communication Systems (VLSICS) Vol.4, No.6, December 2013

Theorem 3. Based on the moduli set $\left\{2^{2 n}, 2^{2 n}+1,2^{2 n}-1\right\}$, the RNS number $\left(x_{1}, x_{2}, x_{3}\right)$ can be converted into its equivalent weighted number $X$ by:

$$
X=m_{1}\left\lfloor\frac{X}{m_{1}}\right\rfloor+x_{1}
$$

where,

$$
\left\lfloor\frac{X}{m_{1}}\right\rfloor=\left|-m_{1} x_{1}-2^{2 n-1} m_{3} x_{2}+2^{2 n-1} m_{2} x_{3}\right|_{m_{2} m_{3}}
$$

Proof. :

From Equation (1), for $k=3$, we obtain:

$$
\begin{aligned}
X= & \left.\left|M_{1}\right| M_{1}^{-1}\right|_{m_{1}} x_{1}+M_{2}\left|M_{2}^{-1}\right|_{m_{2}} x_{2}+ \\
& \left.M_{3}\left|M_{3}^{-1}\right|_{m 3} x_{3}\right|_{m_{1} m_{2} m_{3}}
\end{aligned}
$$

Using Equations (2), (3), and (4) in the above equation, we have:

$$
\begin{aligned}
X= & \mid-m_{2} m_{3} x_{1}+m_{1} m_{3}\left(-2^{2 n-1}\right) x_{2}+ \\
& \left.m_{1} m_{2}\left(2^{2 n-1}\right) x_{3}\right|_{m_{1} m_{2} m_{3}}
\end{aligned}
$$

Substituting Equation (5) into (9) we have:

$$
\begin{aligned}
X= & \mid\left(m_{1}^{2}-1\right)\left(-x_{1}\right)+m_{1} m_{3}\left(-2^{2 n-1}\right) x_{2}+ \\
& \left.m_{1} m_{2}\left(2^{2 n-1}\right) x_{3}\right|_{m_{1} m_{2} m_{3}}
\end{aligned}
$$

Dividing both sides of Equation (10) by $m_{1}$ and computing the floor value of both sides, we obtain:

$$
\left\lfloor\frac{X}{m_{1}}\right\rfloor=\left|-m_{1} x_{1}-2^{2 n-1} m_{3} x_{2}+2^{2 n-1} m_{2} x_{3}\right|_{m_{2} m_{3}}
$$

Equation (11) can therefore be directly rewritten as:

$$
\begin{aligned}
\left\lfloor\frac{X}{2^{2 n}}\right\rfloor= & \mid-2^{2 n} x_{1}-\left(2^{2 n-1}\right)\left(2^{2 n}-1\right) x_{2}+ \\
& \left.2^{2 n-1}\left(2^{2 n}+1\right) x_{3}\right|_{m_{2} m_{3}}
\end{aligned}
$$

Following the basic integer divison definition in RNS, we finally have: 
International Journal of VLSI design \& Communication Systems (VLSICS) Vol.4, No.6, December 2013

$$
\begin{aligned}
X & =m_{1}\left\lfloor\frac{X}{m_{1}}\right\rfloor+x_{1} \\
& =2^{2 n}\left\lfloor\frac{X}{2^{2 n}}\right\rfloor+x_{1}
\end{aligned}
$$

In order to reduce the hardware complexity, we use the following properties to simplify Equation (12):

Property 1 : The multiplication of a residue number by $2^{\mathrm{k}}$ in modulo $\left(2^{\mathrm{P}}-1\right)$ is computed by $\mathrm{k}$ bit circular left shifting

Property 2 : A negative number in modulo $\left(2^{\mathrm{p}}-1\right)$ is calculated by subtracting the number in question from $\left(2^{\mathrm{p}}-1\right)$. In binary representation, the ones complement of the number gives the result.

Let the residues $\left(x_{1}, x_{2}, x_{3}\right)$ have binary representation as follows:

$$
\begin{aligned}
x_{1} & =(\underbrace{x_{1,2 n-1} x_{1,2 n-2} \ldots x_{1,1} x_{1,0}}_{2 n}) \\
x_{2} & =(\underbrace{x_{2,2 n} x_{2,2 n-1} \ldots x_{2,1} x_{2,0}}_{2 n+1}) \\
x_{3} & =(\underbrace{x_{3,2 n-1} x_{3,2 n-2} \ldots x_{3,1} x_{3,0}}_{2 n})
\end{aligned}
$$

Equation (12) can be directly rewritten as :

$$
\left\lfloor\frac{X}{2^{2 n}}\right\rfloor=\left|u_{1}+u_{2}^{\prime}+u_{2}^{\prime \prime}+u_{3}^{\prime \prime \prime}\right|_{2^{4 n}-1}
$$

Where,

$$
\begin{gathered}
u_{1}=\left|-2^{2 n} x_{1}\right|_{2^{4 n-1}}=\underbrace{\bar{x}_{1,2 n-1} \bar{x}_{1,2 n-2} \ldots \bar{x}_{1,0}}_{2 n} \underbrace{11 \ldots 11}_{2 n} \\
u_{2}=\left|-2^{4 n-1} x_{2}+2^{2 n-1} x_{2}\right|_{2^{4 n}-1} \\
u_{2}^{\prime}=\left|-2^{4 n-1} x_{2}\right|_{2^{4 n-1}}=\bar{x}_{2,0} \underbrace{11 \ldots 11}_{2 n-1} \underbrace{\bar{x}_{2,2 n} \bar{x}_{2,2 n-1} \ldots \bar{x}_{2,1}}_{2 n}
\end{gathered}
$$


International Journal of VLSI design \& Communication Systems (VLSICS) Vol.4, No.6, December 2013

$$
\begin{gathered}
u_{2}^{\prime \prime}=\left|2^{2 n-1} x_{2}\right|_{2^{4 n}-1}=\underbrace{x_{2,2 n} x_{2,2 n-1} \ldots x_{2,0}}_{2 n+1} \underbrace{00 \ldots 00}_{2 n-1} \\
u_{3}=\left|2^{4 n-1} x_{3}+2^{2 n-1} x_{3}\right|_{2^{4 n}-1} \\
u_{3}^{\prime}=\left|2^{4 n-1} x_{3}\right|_{2^{4 n-1}}=x_{3,0} \underbrace{00 \ldots 00}_{2 n} \underbrace{x_{3,2 n-1} \ldots x_{3,1}}_{2 n-1} \\
u_{3}^{\prime \prime}=\left|2^{2 n-1} x_{3}\right|_{2^{4 n}-1}=0 \underbrace{x_{3,2 n-1} \ldots x_{3,0}}_{2 n} \underbrace{00 \ldots 00}_{2 n-1}
\end{gathered}
$$

By considering Equation (24) and (25), it is clear that they can be manipulated to obtain $u_{3}^{\prime \prime \prime}$ represented as:

$$
u_{3}^{\prime \prime \prime}=x_{3,0} \underbrace{x_{3,2 n-1} \ldots x_{3,0}}_{2 n} \underbrace{x_{3,2 n-1} \ldots x_{3,1}}_{2 n-1}
$$

\section{HARDWARE REALIZATION}

The hardware structure of the proposed reverse converter for the moduli set $\left\{2^{2 n}, 2^{2 n}+1,2^{2 n}-1\right\}$ is based on Equation $(18)$ and $(14)$. Figure 1 and 2 represents the block diagram of the proposed Cost Efficient (CE) and Speed Efficient (SE) converter respectively . In Equation (14), the parameters $u_{1}, u_{2}^{\prime}, u_{2}^{\prime \prime}$, and $u_{3}^{\prime \prime \prime}$ are added using two $4 n$ bit Carry Save Adders (CSA) with end around carry to produce $s_{2}$ and $c_{2}$. Next, these must be added modulo $2^{4 n}-1$ in order to obtain $\left[\frac{X}{m_{1}}\right]$ to achieve a CE converter. The final result, computed from Equation 14 is therefore obtained simply by a shift and a concatenation operation, which requires no additional hardware resouces. To achieve a SE converter, we speed up the addition process by utilizing anticipated computation. By this, we compute $s_{2}+c_{2}$ for both $\operatorname{cin}=0$ and $\operatorname{cin}=1$ and then the right result is selected with a MUX. Again, the implementation of Equation (14) to obtain the final result requires no additional hardware.

For the sake of completeness, some of the Full Adders (FAs) are reduced to Half Adders (HAs) because some of the inputs of the CSA have constant values of 0 's and 1's. It can be observed that our CE proposal utilizes $10 n F A$ and $2 n H A$ and a conversion time of $(8 n+2) t_{F A}$, while the SE converter requires $10 n F A, 2 n H A$ and $t_{M U X}$, with a delay of $(4 n+2) t_{F A}+t_{M U X}$.

\section{PERFormanCE ANAlysis}

The performance of the proposed reverse converter is evaluated in terms of hardware cost and conversion term. In order to properly evaluate the performance of our proposal against state of the art, both theoretical and experimental analysis are performed. 


\subsection{Theoretical Evaluation}

We compare our converter with state of the art converters presented in [18], [13], and [19]. It must be noted that in [18] and [13], the converters presented are for 5n bit DR moduli sets, while [19] is a 6n bit DR moduli set. The inclusion of [18] and [13] in the comparison is to demonstrate that, our converter can compete favourably with other existing state of the art 5n bit DR moduli sets and for the fact that our proposed moduli set is an improvement of [13]. The theoretical analysis is presented in Table [1]. From the table, it is seen clearly that our proposal outperforms the existing similar dynamic range state of the art converters in terms of area and delay.

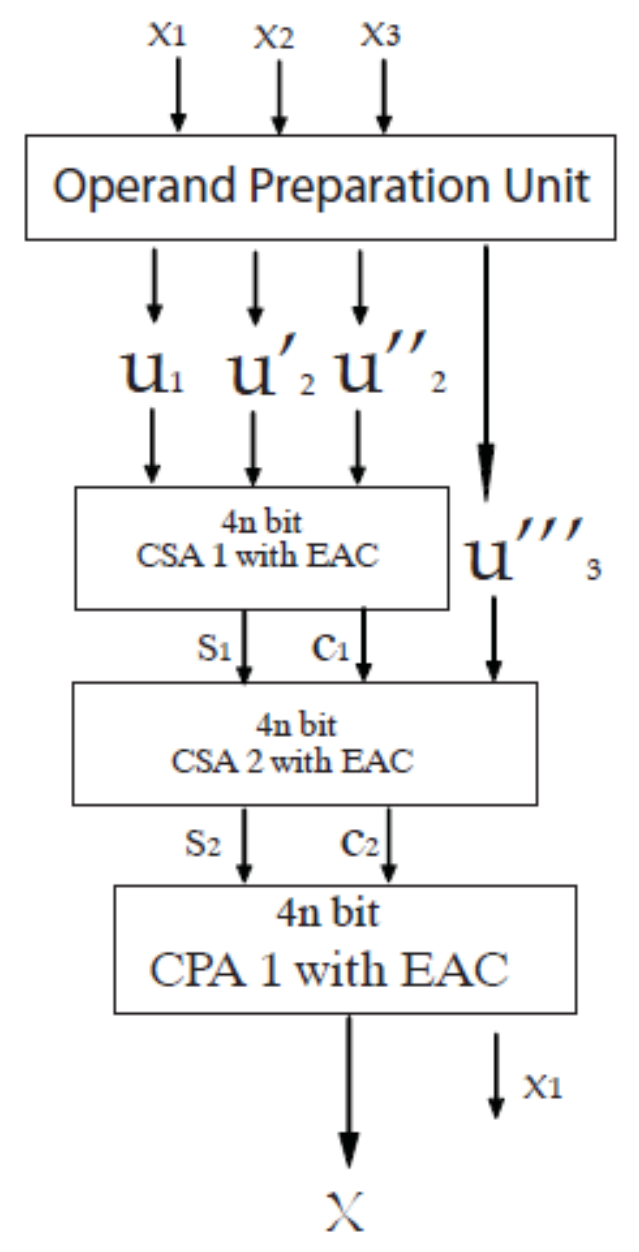

Fig 1. Proposed Cost Efficient Reverse Converter

For our CE converter, the delay is $(8 n+2) t_{\mathrm{FA}}$ while that of [19] exhibits a delay of $(12 n+6) t_{\mathrm{FA}}$. To further simplify the area comparison, we assume that one FA is twice large as an HA, and the expressed the area cost for all the considered designs in terms of HA. It is therefore evident that our converter utilizes lesser area resources. 


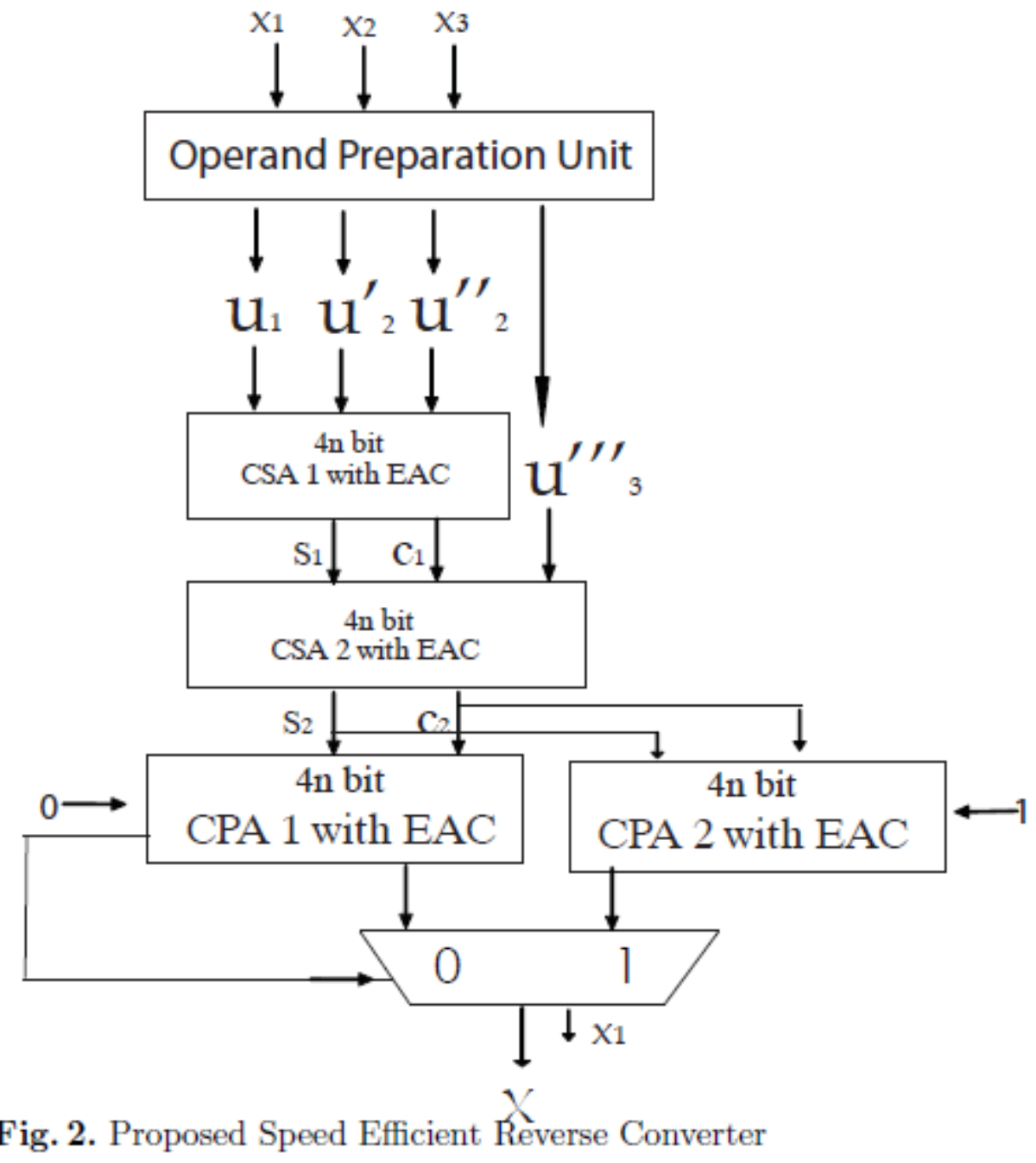

\subsection{Experimental Evaluation}

For the experimental assessment, the converters were described in VHDL and then implemented on Spartan 6 xc6slx45t-3fgg484 FPGA, with Xilinx ISE 14.3 for various dynamic range requirements. The performance is evaluated in terms of area measured according the number of slices and delay corresponding to the critical path in nanoseconds. Table 2 shows the synthesized results for the various values of $n$. To confirm the theoretical results, the experimental results clearly shows the superiority of our converter over the state of the arts. In comparison with the reverse converter presented by [19], the generated values strongly suggest that, on the average, our proposal is capable of performing $52.35 \%$ faster than the converter proposed by [19]. Also, in terms of area cost our converter exhibits a $43.94 \%$ reduction with respect to state of the art. Figures 3 and 4 presents the performance of our proposal against state of the art in terms of delay and area. 
International Journal of VLSI design \& Communication Systems (VLSICS) Vol.4, No.6, December 2013

Table 1. Theoretical Area and Delay Comparison

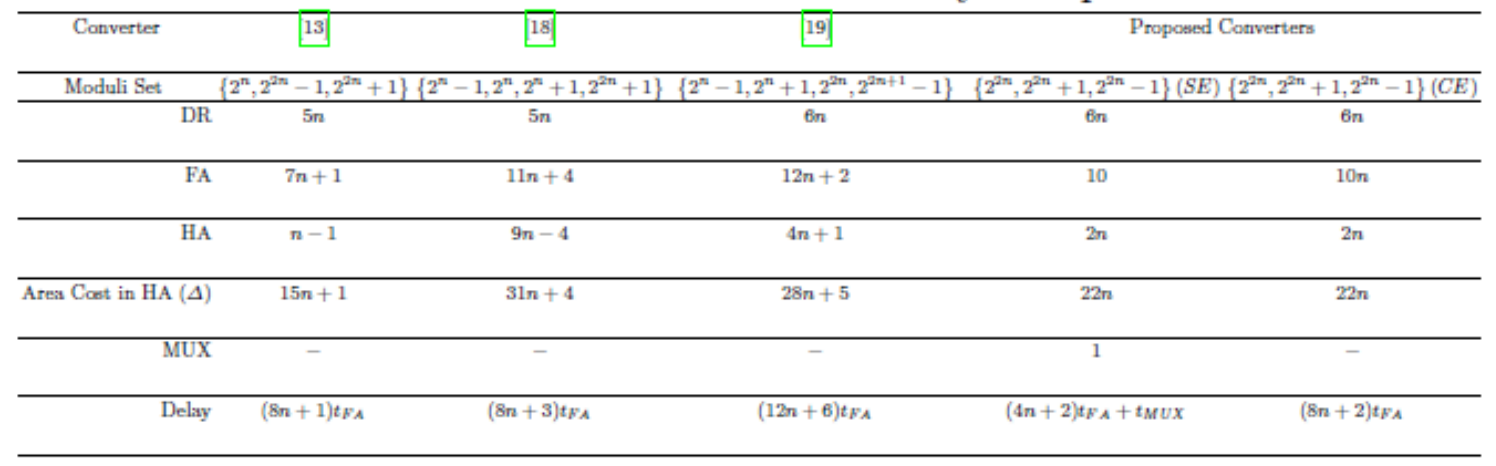

Table 2. Implementation Result : Area, Delay

\begin{tabular}{|l|l|l|l|l|l|l|}
\hline Converter & \multicolumn{2}{|c|}{$[18]$} & \multicolumn{2}{c|}{$[19]$} & \multicolumn{2}{c|}{ Proposed Converter } \\
\hline $\mathrm{n}$ & Delay & Area & Delay & Area & Delay & Area \\
\hline 2 & 18.202 & 54 & 26.650 & 72 & 15.541 & 36 \\
\hline 4 & 22.020 & 107 & 37.011 & 141 & 17.189 & 71 \\
\hline 6 & 26.074 & 157 & 43.637 & 209 & 20.870 & 113 \\
\hline 8 & 24.507 & 210 & 42.755 & 275 & 22.909 & 152 \\
\hline 10 & 26.983 & 270 & 51.522 & 351 & 24.396 & 197 \\
\hline 12 & 26.647 & 327 & 51.892 & 426 & 24.508 & 238 \\
\hline 14 & 27.640 & 386 & 56.011 & 498 & 25.749 & 286 \\
\hline 16 & 28.392 & 440 & 57.778 & 563 & 26.286 & 324 \\
\hline 18 & 29.724 & 489 & 61.110 & 635 & 27.422 & 358 \\
\hline 20 & 29.971 & 546 & 59.663 & 713 & 27.656 & 402 \\
\hline
\end{tabular}

\section{CONCLUSIONS}

In this paper we proposed a novel moduli set $\left\{2^{2 \mathrm{n}}, 2^{2 \mathrm{n}}+1,2^{2 \mathrm{n}}-1\right\}$ with its associated reverse converter using the CRT. The moduli set is a $6 \mathrm{n}$ bit DR and therefore appropriate for applications requiring specifically $6 \mathrm{n}$ DR. We simplified the CRT to obtain an effective algorithm. Further, we reduced the resulting architecture in order to obtain a reverse converter that utilizes only two CSAs and a CPA. We performed both theoretical and experimental evaluation of our proposal. The theoretical analysis shows clearly the advantages of our moduli set and its associated reverse converter. This is confirmed by the experimental results. We described our scheme and those presented by [18] and [19] in VHDL and carried out the implementation on an FPGA using a wide range of values on $\mathrm{n}$. The results indicate that on the average, our scheme is $52.35 \%$ faster than the converter proposed by [19] in terms of speed, while it exhibits a $43.94 \%$ reduction in area cost. Clearly, the results show that, our proposal outperforms the best known state of the art. 
International Journal of VLSI design \& Communication Systems (VLSICS) Vol.4, No.6, December 2013

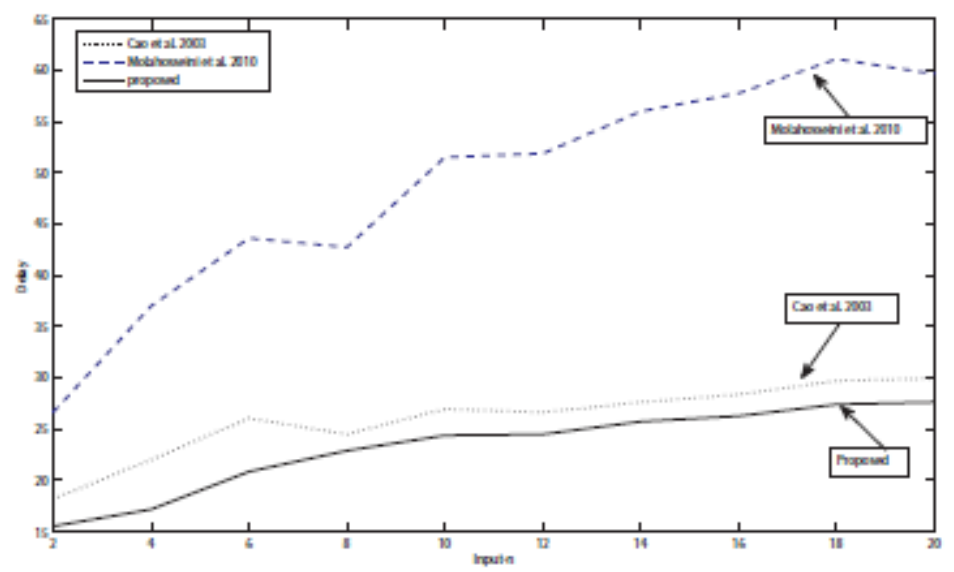

Fig. 3. The Delay Comparison of Converters

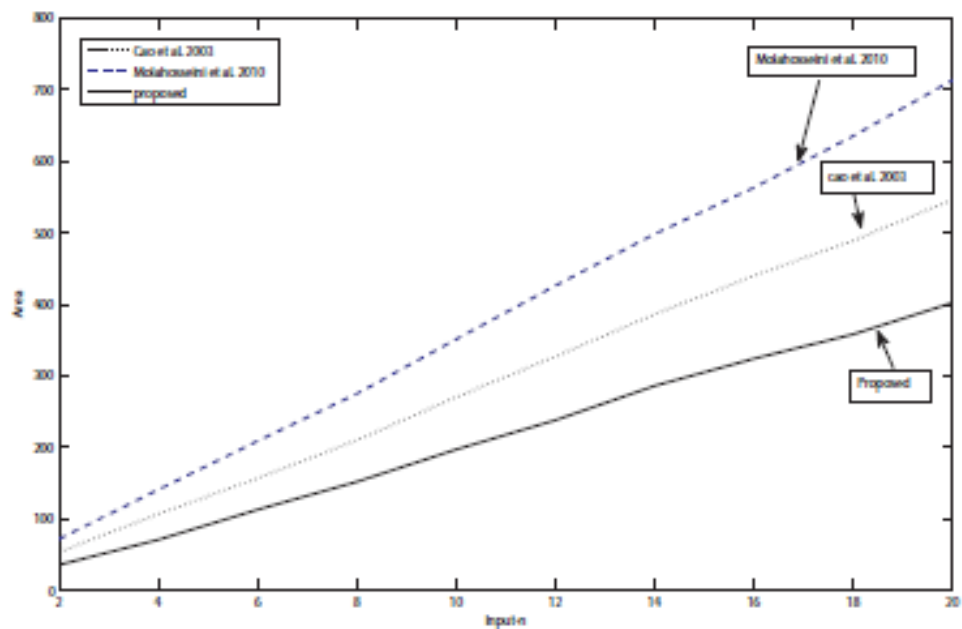

Fig. 4. The Area Comparison of Converters

\section{REFERENCES}

[1] R. Conway and J. Nelson, "Improved RNS FIR filter architectures," IEEE Trans. Circuits Syst. II, vol. 51, no. 1, pp. 26-28, Jan. 2004

[2] N. Szabo and R. Tanaka, Residue Arithmetic and its Application to Computer Technology. MCGraw-Hill, New York, 1967.

[3] M. Bhardwaj, A. Premkumar, and T. Srikanthan, "Breaking the 2n-bit carry propagation barrier in residue to binary conversion for the $\left\{2^{\mathrm{n}}+1,2^{\mathrm{n}}, 2^{\mathrm{n}}-1\right\}$ moduli set." IEEE Trans. on Circuits and Syst. II, vol. 45, pp. 998-1002, September, 1998.

[4] Y. Wang, X. Song, M. Aboulhamid, and H. Shen, "Adder based residue to binary number converters for $\{2 n-1,2 n, 2 n+1\}$." IEEE Trans. on Signal Processing, vol. 50, no. 7, pp. 1772-1779, July, 2002.

[5] E. Bankas and K. Gbolagade, "A speed efficient rns to binary converter for the moduli set $\{2 n, 2 n+1$, 2n-1\}." Journal of Computing, vol. 4, 5, pp. 83-88, 2012.

[6] A. Hiasat and H. Abdel-AtyZohdy, "Residue-to-binary arithmetic converters for the moduli set $\left\{2^{\mathrm{k}}, 2^{\mathrm{k}}\right.$ - 1, $\left.2^{\mathrm{k}-1}-1\right\}$," IEEE Transactions on Circuits and Systems-II. Analog and Digital Signal Processing, vol. 45, no. 2, pp. 204-209, Feb., 1998. 
[7] M. Hosseinzadeh, A. Molahosseini, and K. Navi, "A parallel implementation of the reverse converter for the moduli set $\left\{2^{n}, 2^{n}-1,2^{n-1}-1\right\}$." World Academy of Sciences, Engineering and Technology, vol. 55, pp. 494-498, July, 2009.

[8] S. Lin, M. Sheu, and C. Wang, "Efficient vlsi design of residue-to-binary converter for the moduli set $\left\{2^{\mathrm{n}}, 2^{\mathrm{n}+1}-1,2^{\mathrm{n}}-1\right\}$."IEICE Trans. Info and Systems, vol.E91-D, no. 7, pp. 2058-2060, 2008.

[9] A. Mohan, "Rns to-binary converters for a new three moduli set $\left\{2^{n+1}-1,2^{n}, 2^{n}-1\right\}$."IEEE Trans. on Circuits and Systems-II: Express briefs, vol. 54, no. 9, pp. 775-779, Sep., 2007.

[10] K. Gbolagade, R. Chaves, L. Sousa, and S. Cotofana, "An improved rns reverse converter for the $\left\{2^{2 n+1}-1,2^{n}, 2^{n}-1\right\}$ moduli set ." IEEE International Symposium on Circuits and Systems (ISCAS 2010), Paris, France, pp. 2103-2106, 2010.

[11] K. Navi, M. Esmaeildoust, and A. Molahosseini, "A general reverse converter architecture with low complexity and high performance," IEICE Trans. Inf. and System., vol. E94-D,, vol. E94-D, pp. 264273, 2011.

[12] E. Bankas, K. Gbolagade, and S. Cotofana, "An effective new crt based converter for a novel moduli set $\{22 n+1-1,22 n+1,22 n-1\}, " 24$ th IEEE International Conference on Application-specific Systems, Architectures and processors (ASAP 2013), pp. 142-146, June, 2013.

[13] A. Hariri, K. Navi, and R. Rastegar, "A new high dynamic range moduli set with efficient reverse converter," Computers and Mathematics with Applications, vol. 55, pp. 660-668, 2008.

[14] K. Gbolagade and S. Cotofana, "An efficient RNS to binary converter using the moduli set $\{2 n+1$, 2n, 2n - 1\}," in XXIII Conf. Design of Circuits and Integrated Systems (DCIS 2008), Grenoble, France, Nov. 2008.

[15] A. Premkumar, "An RNS to binary converter in a three moduli set with common factors," IEEE Trans. Circuits Syst. II, vol. 42, no. 4, pp. 298-301, Apr 1995.

[16] B. Vinnakota and V. Rao, "Fast conversion techniques for binary-residue number systems," IEEE Trans. Circuits Syst. I, vol. 41, pp. 927-929, Dec. 1994.

[17] M. Akkal and P. Siy, "A new mixed radix conversion algorithm MRC-II," J. Syst. Archit., vol. 53, pp. 577-586, 2007.

[18] B. Cao, C. Chang, and T. Srikanthan, "An efficient reverse converter for the 4-moduli set $\left\{2^{n}-1,2^{n}\right.$, $2^{\mathrm{n}}+1,2^{2 \mathrm{n}}+1$ based on the new chinese remainder theorem." IEEE Trans. on Circuits and Systems-I: Fundamental Theory and Applications, vol. 50, no. 10, pp. 1296-1303, Oct., 2003.

[19] A. Molahosseini and K. Navi, "A reverse converter for the enhanced moduli set $\left\{2^{\mathrm{n}}-1,2^{\mathrm{n}}+1,2^{2 \mathrm{n}}\right.$, $\left.2^{2 n+1}-1\right\}$ using crt and mrc." IEEE Annual Symposium on VLSI., pp. 456-457, 2010.

[20] W. Wang, M. Swamy, M. Ahmad, and Y. Wang, "A study of the residue-to-binary converters for the three-moduli sets," IEEE Trans. Circuits Syst. I, vol. 50, no. 2, pp. 235-243, Feb. 2003.

[21] M. Ahmad, Y. Wang, and M. Swamy, "Residue-to-binary number converters for three moduli sets," vol. 46, no. 7, Feb 1999, pp. 180-183.

[22] A. Premkumar, "An RNS to binary converter in $\{2 n+1,2 n, 2 n-1\}$ moduli set,"IEEE Trans. Circuits Syst. II, vol. 39, no. 7, pp. 480-482, July 1992.

[23] Y. Wang, "Residue-to-binary converters based on new chinese remainder theorems," IEEE Trans. Circuits Syst. II, vol. 47, no. 3, pp. 197-205, Mar. 2000.

[24] S. Andraos and F. Ahmad, "A new efficient memoryless residue to binary converter." IEEE Trans. on Circuits and Systems-II., vol. 35, no. 11, pp. 1441-1444, Nov., 1988.

[25] S. Piestrak, "A high-speed realization of a residue to binary number system converter." IEEE Trans. on Circuits and Systems-II: Analog and Digital Signal Processing, vol. 42, no. 10, pp. 661-663, Oct., 1995. 